\title{
ON OPTIMAL CANCELLATION OF POLICIES
}

\author{
HANS U. GERBER
}

\section{INTRODUCTION}

One of the basic problems in life is: Given information (from the past), make decisions (that will affect the future). One of the classical actuarial examples is the adaptive ratemaking (or credibility) procedures; here the premium of a given risk is sequentially adjusted, taking into account the claims experience available when the decisions are made.

In some cases, the rates are fixed and the premiums cannot be adjusted. Then the actuary faces the question: Should a given risk be underwritten in the first place, and if yes, what is the criterion (in terms of claims performance) for cancellation of the policy at a later time?

Recently, Cozzolino and Freifelder [6] developed a model in an attempt to answer these questions. They assumed a discrete time, finite horizon, Poisson model. While the results lend themselves to straightforward numerical evaluation, their analytical form is not too attractive. Here we shall present a continuous time, infinite horizon, diffusion model. At the expense of being somewhat less realistic, this model is very appealing from an analytical point of view.

Mathematically, the cancellation of policies amounts to an optimal stopping problem, see [8], [4], or chapter 13 in [7], and (more generally) should be viewed within the framework of discounted dynamic programming [I], [2].

\section{A Differential Equation and its Solutions}

Our model will turn out to be very tractable because the differential equation

$$
[x(\mathrm{I}-x)]^{2} W^{\prime \prime}(x)=\alpha W(x), \quad \alpha>0
$$

can be solved explicitly. Observe that this differential equation 
has (regular) singular points at $x=0$ and $x=\mathrm{r}$. The reader will easily verify that

$$
h(x)=\frac{(1-x)^{c}}{x^{c-1}}
$$

is a solution, where $c>I$ is the positive solution of $c(c-I)=x$, i.e.

$$
c=\frac{1}{2}+\frac{1}{2} \sqrt{I+4 \alpha}
$$

For reasons of symmetry, also $h(I-x)$ is a solution. Thus every solution of equation $(I)$ is a linear combination of $h(x)$ and $h(\mathrm{I}-x)$. Equation $\left(\mathrm{I}^{\prime}\right)$ of Section 5 will be of more general form but can also be solved by a function of the type (2).

\section{INDEPENDENT RISKS}

In this section we assume that the income processes resulting from different policies are independent. Therefore we can restrict ourselves to the discussion of a single policy.

We shall suppose that the quality of a given risk is determined by a well defined, but not directly observable random variable $\theta$ (the risk parameter). Let $X_{t}$ denote the aggregate gain that is generated by the policy from $o$ to $t$. Then we assume that, for given $\theta$,

$$
X_{t}=(r \theta-a) t+\sigma W_{t}
$$

Here $r, a, \sigma$ are positive constants, and $\left\{W_{t}\right\}$ is the standard Wiener process (independent of $\theta$ ). Having observed the aggregate gains, we will be interested in the posteriori distribution of $\theta$. The discussion of this will be greatly simplified by our assumption that $\theta$ has only the values $O$ or $I$. So let

$$
\pi=P[\theta=\mathrm{I}], \mathrm{I}-\pi=P[\theta=0]
$$

be the priori probabilities (at time o), and

$$
\pi_{t}=P_{\pi}\left[\theta=\mathrm{I} \mid X_{u}, 0 \leq u \leq t\right]
$$

denote the posteriori probabilities (which depend on the priori probability as well as on the observed profitability of the policy). To make things interesting, we assume that $r>a$. Thus if $\theta=\mathrm{I}$, our policy is a "good" risk; if $\theta=0$, it is a "bad" risk (at least as far as expected gains are concerned). 
Let $\delta>0$ be a constant force of interest. The insurer's decision is now the selection of a stopping rule $T$; for every $\pi, 0 \leq \pi \leq I$, $T=T(\pi)$ thereby defines a possibly defective stopping time. We interpret $T$ as the time when the policy is cancelled, with the provision that the policy will not be cancelled if $T=\infty$. Let

$$
V(\pi ; T)=E_{\pi}\left[\int_{0}^{T} e^{-\delta t} d X_{t}\right]
$$

denote the expected present value of the total gain. If we extend the integral to infinity, and subtract the correction term, we obtain an alternative definition:

$$
V(\pi ; T)=\frac{\pi r-a}{\delta}-E_{\pi}\left[\frac{\pi T^{r}-a}{\delta} e^{-\delta T}\right]
$$

The problem is now to find an optimal stopping rule $T$, i.e. one that maximizes $V(\pi ; T)$ for every $\pi$, or equivalently, one such that

$$
E_{\pi}\left[\frac{a-\pi_{T^{r}}}{\delta} e^{-\delta T}\right]
$$

is maximal.

The process $\left\{\pi_{t}\right\}$ is a diffusion process with vanishing drift and infinitesimal variance

$$
\sigma^{2}(\pi)=\frac{r^{2}}{\sigma^{2}}[\pi(I-\pi)]^{2}
$$

For a sophisticated proof of this, see Lemma 5 of Chapter 4 in [8]. A more heuristic derivation goes as follows: For given $X_{u}$, $0 \leq \imath \leq t, X_{t}$ is a sufficient statistic. Therefore

$$
\pi_{t}=\frac{\pi n\left(X_{t} ; r t-a t, \sigma^{2} t\right)}{\pi n\left(X_{t} ; r t-a t, \sigma^{2} t\right)+(I-\pi) n\left(X_{t} ;-a t, \sigma^{2} t\right)}
$$

where $n\left(. ; \mu, \sigma^{2}\right)$ denotes the normal density with mean $\mu$ and variance $\sigma^{2}$. This can be simplified to

$$
\pi_{t}=f\left(\pi, X_{t}, t\right)
$$

where

$$
f(\pi, x, t)=\frac{\pi}{\pi+(I-\pi) g(x, t)}
$$


and

$$
g(x, t)=\exp \left[-\frac{r}{\sigma^{2}}\left(x-\frac{r}{2} t+a t\right)\right]
$$

Since $\left\{\pi_{t}\right\}$ is a Markov process (posteriori probabilities always are) and can be expressed as a well behaved function of $X_{t}$, see (I2), it has to be a diffusion process, say with drift $\mu(\pi)$ and infinitesimal variance $\sigma^{2}(\pi)$. A Taylor series argument shows that

$$
\begin{aligned}
& \mu(\pi)=(r \pi-a) \frac{\partial f}{\partial x}+\frac{\sigma^{2}}{2} \frac{\partial^{2} f}{\partial x^{2}}+\frac{\partial f}{\partial t}=0 \\
& \sigma^{2}(\pi)=\sigma^{2}\left[\frac{\partial f}{\partial x}\right]^{2}=\frac{r^{2}}{\sigma^{2}}[\pi(I-\pi)]^{2}
\end{aligned}
$$

(the argument in the partial derivatives is $x=t=0$ ). Of course we could have anticipated the vanishing drift: Posteriori probabilities always constitute a martingale (law of total probability)!

Let us introduce the function $V(\pi)$,

$$
V(\pi)=\underset{T}{\operatorname{supremum}} V(\pi ; T)
$$

Then an optimal stopping rule $T$ is given by the formula

$$
T=\left\{\begin{array}{l}
\infty \text { if } V\left(\pi_{t}\right)>0 \text { for } t \geq 0 \\
\text { inf }\left\{t \mid V\left(\pi_{t}\right)=0\right\} \text { otherwise }
\end{array}\right.
$$

Obviously $V(\pi)$ is a nondecreasing function. Therefore the set of numbers $\pi$ such that $V(\pi)>0$ is an interval $(p, I]$. Hence we can restrict ourselves to stopping rules of the form

$$
T_{p}=\inf \left\{t \mid \pi_{t} \leq p\right\}
$$

Our initial problem is now reduced to the discussion of the function $V(\pi, p)=V\left(\pi ; T_{p}\right), 0 \leq p \leq \pi \leq \mathrm{I}$, and to the search for the optimal value of $p$, call it $p_{0}$. Formula (8) reduces in this case to

$$
V(\pi, p)=\frac{\pi r-a}{\delta}-\frac{p r-a}{\delta} W(\pi, p)
$$

where

$$
W(\pi, p)=E_{\pi}\left[e^{-\delta T_{p}}\right]
$$


is the present value of a unit payable at the time when $\pi_{t}=p$. Furthermore it is clear that the policy should not be cancelled as long as $\pi_{i} r-a>0$. Therefore we expect that $p_{0} \leq a / r$.

It is well known (see for example Problem I9, Chapter I6 of [3]) that the function $W(\pi, p)$ satisfies the differential equation

$$
\frac{1}{2} \sigma^{2}(\pi) \frac{\partial^{2} W}{\partial \pi^{2}}-\delta W=0
$$

valid for $p<\pi<\mathrm{I}$, where $\sigma^{2}(\pi)$ is given by formula (IO). (For a short derivation of this equation, observe that the process $\left\{e^{-\delta t} W\left(\pi_{t}, p\right)\right\}, t \leq T_{p}$, is a martingale). Obviously, the function $W$ is continuous in the closed interval $p \leq \pi \leq I$ and satisfies the boundary conditions

$$
W(p, p)=I, \quad W(\mathrm{I}, p)=0 .
$$

By recalling the results of Section 2, we find that the solution of conditions (22) and (23) is

$$
W(\pi, p)=\frac{h(\pi)}{h(p)}, p \leq \pi \leq \mathrm{I}
$$

where $h(x)$ is given by formula (2) with parameter

$$
c=\frac{1}{2}+\frac{1}{2} \sqrt{I+8 \delta \sigma^{2} / r^{2}}
$$

Thus formula (20) becomes

$$
V(\pi, p)=\frac{\pi r-a}{\delta}-\frac{p r-a}{\delta} \frac{(I-\pi)^{c}}{\pi^{c-1}} \frac{p^{c-1}}{(I-p)^{c}}
$$

valid for $p \leq \pi \leq I$, and we are looking for the value of $p$ that maximizes

$$
(a-p r) \frac{p^{c-1}}{(I-p)^{c}}
$$

Differentiation leads to the optimal value:

$$
p_{0}=b\left(\frac{c-I}{c-b}\right)
$$

where $b=a / r$ and $c$ is given by formula (25). 


\section{Remark I}

If we let the force of interest increase from 0 to $\infty$ (and keep the other parameters constant), $c$ increases from $I$ to $\infty$, and therefore $p_{0}$ increases from 0 to $b$. This is not surprising: The smaller the rate of interest is, the more it pays off to postpone the cancellation of the policy, hoping to obtain more reliable information about the quality of the risk in the future.

\section{Remark 2}

For an arbitrary $p$, we obtain from formula (26) that

$$
\left.\frac{\partial V}{\partial \pi}\right|_{\pi=p}=\frac{r}{\delta} \frac{(c-b)\left(p-p_{0}\right)}{p(\mathrm{I}-p)}
$$

Thus the right side derivative at $\pi=p$ is positive (negative) if $p>p_{0}\left(p<p_{0}\right)$ and zero for $p=p_{0}$. Smooth pasting conditions of this kind hold in more general models, see Section 6 , Chapter 3 of [8].

\section{Remark 3}

Formulae (I2), (I3), (I4) allow us to express the stopping rule $T_{p}$ in terms of $\left\{X_{t}\right\}$ and $\pi$. Let $K=K(\pi, p)$ be the solution of

Then

$$
\frac{\pi}{\pi+(\mathrm{I}-\pi) e^{r k / \sigma^{2}}}=p
$$

$$
T_{p}=\inf \left\{t \mid X_{t} \leq(r / 2-a) t-K\right\}
$$

is equivalent to the original definition (Ig).

\section{Discussion of the Time of Cancellation}

The function $W(\pi, p)$ can be interpreted as the Laplace transform of $T_{p}$ (for given $\pi$ and $p$ ):

$$
\phi(\delta)=E_{\pi}\left[e^{\left.-\delta T_{p}\right]}=\frac{(\mathrm{I}-\pi)^{c}}{\pi^{c-1}} \frac{p^{c-1}}{(\mathrm{I}-p)^{c}}\right.
$$

where $c=c(\delta)$ is given by formula (25). Thus the probability for cancellation of the policy is

$$
\phi(0)=P_{\pi}\left(T_{p}<\infty\right)=\frac{I-\pi}{I-p}
$$


If $\theta=0$, the policy will be cancelled with probability one. Therefore the probability for $\theta=I$ and cancellation (i.e. "erroneous" cancellation) is

$$
\frac{\mathrm{I}-\pi}{\mathrm{I}-\mathrm{p}}-(\mathrm{I}-\pi)=\frac{p}{\mathrm{I}-\mathrm{p}}(\mathrm{I}-\pi)
$$

and the conditional probability for cancellation, given that $\theta=I$, is

$$
\frac{p}{I-p} \frac{I-\pi}{\pi}
$$

Finally, we are interested in the distribution of the time of cancellation, given that it occurs. Let $\underset{\psi}{\mathrm{u}}(\delta)$ denote the Laplace transform of this proper distribution. Thus

$$
\psi(\delta)=\phi(\delta) / \phi(0)=e^{-m(c-1)}
$$

where $m=m(\pi, p)$ is given by the formula

$$
m=\ln \frac{I / P-I}{I / \pi-I}
$$

We recognize that the distribution of $T_{p}$ (given $T_{p}<\infty$ ) is infinitely divisible. Its first two moments are:

$-\psi^{\prime}(0)=E_{\pi}\left[T_{p} \mid T_{p}<\infty\right]=\frac{2 \sigma^{2}}{r^{2}} m(\pi, p)$

$$
\psi^{\prime \prime}(0)-\psi^{\prime}(0)^{2}=\operatorname{Var}_{\pi}\left[T_{p} \mid T_{p}<\infty\right]=\frac{8 \sigma^{4}}{r^{4}} m(\pi, p)
$$

Moreover, formula (36) can be inverted. The underlying density, say $g(t), t>0$, is

$$
g(t)=\frac{m \sigma}{r \cdot \sqrt{2 \pi}} t^{-3 / 2} \exp \left\{-\frac{r^{2}}{\sigma^{2}} \frac{\left(\frac{t}{2}-m \frac{\sigma^{2}}{r^{2}}\right)^{2}}{2 t}\right\}
$$

This can be seen by a comparison with formulas (73) and (75), Section 5.7 in [5], or from problem $I_{4}$, p. +39 in [9].

\section{LAPSES}

In this section we modify the model of Section 3 and allow for the possibility of termination of the policy by the insured. For 
simplicity we assume that the time of termination by the insured, say $S$, is (for given $\theta$ ) exponentially distributed but otherwise independent of $\left\{X_{t}\right\}$.

$$
P_{\pi}[S>t]=\pi e^{-\lambda_{1} t}+(\mathrm{I}-\pi) e^{-\lambda_{0} t}
$$

Here $\lambda_{I}>0$ is the constant force of lapse of the "good" risks, $\lambda_{0}>0$ the one of the "bad" risks.

The insurer is only interested in times $t<S$. Therefore we investigate

$$
\bar{\pi}_{t}=P_{n}\left[\theta=\mathrm{I} \mid X_{u}(0 \leq u \leq t), S>t\right]
$$

Again $\pi_{t}$ is of the form (12), with $f$ defined as in formula (I3) where $g$ is now

$$
g(x, t)=\exp \left[-\frac{r}{\sigma^{2}}\left(x-\frac{r}{2} t+a t\right)+\left(\lambda_{1}-\lambda_{0}\right) t\right]
$$

By the same arguments as in Section 3 we recognize that $\left\{\pi_{t}\right\}$ is a diffusion process with drift

$$
\mu(\pi)=\left(\lambda_{0}-\lambda_{1}\right) \pi(I-\pi)
$$

and infinitesimal variance $\sigma^{2}(\pi)$ as in formula (I6).

We want to maximize

$$
V(\pi ; T)=E_{\pi}\left[\int_{0}^{310(s, r)} e^{-\delta t} d X_{t}\right]
$$

and may restrict ourselves to cancellation times $T_{p}$ of the form

$$
T_{p}=\operatorname{Min}\left\{t \mid \pi_{t} \leq p \text { or } S=t\right\}
$$

For $p \leq \pi \leq \mathrm{I}$, its value $V(\pi, p)$ is

$$
\begin{aligned}
V(\pi, p) & =\frac{\pi(r-a)}{\delta+\lambda_{1}}-\frac{(I-\pi) a}{\delta+\lambda_{0}} \\
& +\left\{\frac{p(r-a)}{\delta+\lambda_{1}}-\frac{(I-p) a}{\delta+\lambda_{0}}\right\} W(\pi, p)
\end{aligned}
$$

where

$$
W(\pi, p)=E_{\pi}\left[e^{-\delta T_{p}} \mid S>T_{p}\right] P_{\pi}\left[S>T_{p}\right]
$$

Using the facts that $\left\{\pi_{t}\right\}$ is a diffusion process and that

$$
\left\{e^{-\delta t}\left[\pi e^{-\lambda_{1} t}+(I-\pi) e^{-\lambda_{\theta} t}\right] W\left(\pi_{t}, p\right)\right\}
$$


is a martingale for $\pi_{t}>p$, we see that $W(\pi, p)$ satisfies the differential equation

$\frac{1}{2} \sigma^{2}(\pi) \frac{\partial^{2} W}{\partial \pi^{2}}+\mu(\pi) \frac{\partial W}{\partial \pi}-\left[\delta+\pi \lambda_{1}+(\mathrm{I}-\pi) \lambda_{0}\right] W=0$

valid for $p<\pi<I$, where $\sigma^{2}(\pi)$ and $\mu(\pi)$ are given by formulas (I6) and $\left(15^{\prime}\right)$. The boundary conditions

$$
W(p, p)=\mathrm{I}, \quad W(\mathrm{I}, p)=0
$$

are obvious.

Luckily, a differential equation of the form

$$
\begin{aligned}
\frac{r^{2}}{2 \sigma^{2}}[x(\mathrm{I}-x)]^{2} W^{\prime \prime}+\left(\lambda_{0}-\lambda_{1}\right) & x(\mathrm{I}-x) W^{\prime}= \\
& {\left[\delta+x \lambda_{1}+(\mathrm{I}-x) \lambda_{0}\right] W }
\end{aligned}
$$

can be solved explicitly. The solution that vanishes at $x=\mathrm{I}$ is a multiple of the function $h(x)$, see formula (2), whose parameter $c$ is the positive root of the equation

$$
\frac{r^{2}}{2 \sigma^{2}} c(c-I)+\left(\lambda_{1}-\lambda_{0}\right)(c-I)-\left(\delta+\lambda_{0}\right)=0
$$

i.e.

$$
\begin{aligned}
c=\frac{1}{2}+ & \frac{\sigma^{2}}{r^{2}}\left(\lambda_{0}-\lambda_{1}\right)+ \\
& +\frac{1}{2} \sqrt{\left[\mathrm{I}+\frac{2 \sigma^{2}}{r^{2}}\left(\lambda_{0}-\lambda_{1}\right)\right]^{2}+\frac{8 \sigma^{2}}{r^{2}}\left(\delta+\lambda_{1}\right)}
\end{aligned}
$$

Observe that $c>I$.

From this and conditions $\left(22^{\prime}\right)$ and $\left(23^{\prime}\right)$ we see that

$$
W(\pi, p)=\frac{h(\pi)}{h(p)}, p \leq \pi \leq \mathrm{I},
$$

which then can be substituted in formula $\left(20^{\prime}\right)$. The optimal value of $p$, say $p_{0}$, is therefore the value of $p$ that maximizes

$$
\left\{\frac{(\mathrm{I}-p) a}{\delta+\lambda_{0}}-\frac{p(r-a)}{\delta+\lambda_{1}}\right\} \frac{p^{c-1}}{(\mathrm{I}-p)^{c}}
$$


Thus

$$
p_{0}=b \frac{c-I}{(c-b)+c(I-b) \frac{\lambda_{0}-\lambda_{1}}{\delta+\lambda_{1}}}
$$

as can be seen by differentiation.

\section{ILI.USTRATION}

The effect of lapses is illustrated in Tables I and 2 . The parameter $c$ and $p_{0}$ (the optimal value of $p$ ) were computed for nine combinations $\left(\lambda_{0}, \lambda_{1}\right)$. Thereby the other parameters of the model were kept fixed, namely $a=\mathrm{I}, r=2, b=.5, \sigma=2, \delta=. \mathrm{I}$. A glance at Table 2 shows that the $p_{0}$-values decrease in each row as $\lambda_{0}$ increases. The explanation for this is: The higher the lapse rates of the bad risk, the better this is for the insurer. On the other hand, the po-values increase in each column (as $\lambda_{1}$ increases): The higher the lapse rates of the good risks, the worse this is for the insurer. Finally, the $p_{0}$-values in the main diagonal are increasing: If $\lambda_{0}=\lambda_{1}=\lambda$, lapses simply amount to an increased force of interest, $\bar{\delta}=\delta+\lambda$, and we know that $p_{0}$ is an increasing function of the interest rate (see remark $I$ at the end of Section 3). In any case the $p_{0}$-value is well below $b=\frac{1}{2}$ : For prior probabilities $\pi$ with $p_{0}<\pi<\frac{1}{2}$ it pays off to postpone cancellation and to suffer an expected loss of $I-2 \pi$ per unit time in the nearest future.

TABLE I

Values of the parameter $c$

\begin{tabular}{llll}
\hline$\lambda_{1}$ & 0 & .1 & .2 \\
\hline 0 & 1.171 & 1.348 & $1.53 I$ \\
.$I$ & 1.148 & 1.307 & 1.472 \\
.2 & $1.13 I$ & 1.272 & 1.422 \\
\hline
\end{tabular}


TABLE 2

Optimal values of $p$

\begin{tabular}{llll}
\hline$\lambda_{1}$ & 0 & .1 & .2 \\
\hline 0 & .127 & $.1 I_{4}$ & .104 \\
.$I$ & .205 & .190 & .176 \\
.2 & .257 & .243 & .229 \\
\hline
\end{tabular}

\section{DEPENDENT RISKS}

We shall consider only the most simple case, namely the case of two dependent risks. Supposedly we observe the aggregate gains $X_{t}^{1}, X_{t}^{2}$ of two policies, which can be represented as follows:

$$
\begin{aligned}
& X_{t}^{1}=(r \theta-a) t+\sigma W_{t}^{1} \\
& X_{t}^{2}=[r(\mathrm{I}-\theta)-a] t+\sigma W_{t}^{2}
\end{aligned}
$$

Here $\left\{W_{t}^{i}\right\}$ denote standard Wiener processes, and $\theta,\left\{W_{t}^{1}\right\},\left\{W_{t}^{2}\right\}$ are assumed to be independent. Again, let $\sigma>0,0<a<r$. The random variable $\theta$ assumes the values 0 or $I$ and specifies which policy constitutes the "good" risk: If $\theta=I$, the gain of policy I has drift $r-a>0$ and the gain of policy 2 has gain $-r$; if $\theta=0$, the roles are interchanged.

Let $\pi=P[\theta=\mathrm{I}]$ and

$$
\pi_{t}=P_{\pi}\left[\theta=\mathrm{I} \mid X_{u}^{1}, X_{u}^{2} \text { for } 0 \leq u \leq t\right]
$$

At time $t, X_{t}^{1}-X_{t}^{2}$ is a sufficient statistic for $\theta$. From this we get that

$$
\pi_{t}=f\left(\pi, X_{t}^{1}-X_{t}^{2}\right)
$$

where

$$
f(\pi, x)=\frac{\pi}{\pi+(\mathrm{I}-\pi) g(x)}
$$

with

$$
g(x)=e^{-r x / \sigma^{2}}
$$


Thus the process $\left\{\pi_{t}\right\}$ is a diffusion process, namely with vanishing drift and infinitesimal variance

$$
\sigma^{2}(\pi)=2 \sigma^{2}\left(\left.\frac{\partial f}{\partial x}\right|_{x=0}\right)^{2}=\frac{2 \gamma^{2}}{\sigma^{2}}[\pi(\mathrm{I}-\pi)]^{2}
$$

Observe that this is just twice the infinitesimal variance that would be effective if we could observe the gains process of only one policy, see formula (IO).

Cancellation rules $T$ are defined as in Section 3. Of special interest is the family of rules $T_{q}$ such that (for $0<q \leq \frac{1}{2}$ )

$$
T_{q}=\operatorname{minimum}\left\{t / \pi_{t} \leq q \text { or } \pi_{t} \geq \mathrm{I}-q\right\}
$$

with the understanding that we cancel policy $I$ if $\pi_{T_{q}} \leq q$, but that we cancel policy 2 if $\pi T_{q} \geq I-q$. We shall restrict ourselves to cancellation rules of this type.

\section{a) Variant I: Only one cancellation}

Here we allow for the cancellation of one policy only. If after the cancellation it turns out that we made the wrong decision the other policy cannot be cancelled.

Let $\bar{V}(\pi, q)$ be the value of $T_{q}$. Obviously

$$
\bar{V}(\pi, q)= \begin{cases}\frac{(I-\pi) r-a}{\delta} & \text { for } 0 \leq \pi \leq q \\ \frac{\pi r-a}{\delta} & \text { for } \quad I-q \leq \pi \leq \mathrm{I} .\end{cases}
$$

As long as both policies are in force, their total gain has drift $r-2 a$. Therefore, for $q<\pi<I-q$,

$$
\bar{V}(\pi, q)=\frac{r-2 a}{\delta}-\frac{q r-a}{\delta} \bar{W}(\pi, q)
$$

where

$$
\bar{W}(\pi, q)=E_{\pi}\left[e^{-\delta T_{q}}\right]
$$

Since $\left\{e^{-\delta t} \bar{W}\left(\pi_{t}, q\right)\right\}, t<T_{q}$, is a martingale, the function $\bar{W}$ satisfies the differential equation

$$
\frac{1}{2} \sigma^{2}(\pi) \frac{\partial 2 \bar{W}}{\partial \pi^{2}}-\delta \bar{W}=0, \quad q<\pi<I-q
$$


subject to the boundary conditions

$$
\bar{W}(q, q)=\bar{W}(\mathrm{I}-q, q)=\mathrm{I}
$$

Recalling formula (49) and the results of Section 2 we gather that

$$
\bar{W}(\pi, q)=\frac{h(\pi)+h(\mathrm{I}-\pi)}{h(q)+h(\mathrm{I}-q)}
$$

where the parameter of $h(x)$ is now

$$
c=\frac{1}{2}+\frac{1}{2} \sqrt{I+4 \delta \sigma^{2} / r^{2}}
$$

Substituting the above expression for $\bar{W}$ in formula (52), we recognize that we should choose $q$ in order to maximize the quantity

$$
(a-q r)\left[\frac{(\mathrm{I}-q)^{c}}{q^{c-1}}+\frac{q^{c}}{(\mathrm{I}-q)^{c-1}}\right]^{-1}
$$

b) Variant 2: Possibly two cancellations

If we have the option to cancel the second policy, we will cancel it according to the optimal rule that was established in Section 3.

Now the value of $T_{q}$, say $\overline{\bar{V}}(\pi, q)$, is

$$
\overline{\bar{V}}(\pi, q)=\frac{r-2 a}{\delta}+\left[-\frac{r-2 a}{\delta}+V\left(\mathrm{I}-q, p_{0}\right)\right] \bar{V}(\pi, q)
$$

valid for $q \leq \pi \leq \mathrm{I}-q$.

\section{ACKNOWLEDGEMENT}

Research for this paper was supported by a Faculty Research Fellowship of the University of Michigan.

\section{REFERENCES}

[l] Blackwell, D., "Discounted dynamic programming," Ann. Math. Statist. 36, 226-235 (1965).

[2] Breiman, L., "Stopping-rule problems," Applied Combinatorial Mathematics (ed. by Beckenbach), 284-3I9, Wiley, New York (I964).

[3] Breiman, L., Probability, Addison-Wesley, Reading (I968).

[4] Chow, Y. S., RobBins, H. and Siegmund, D., Great expectations: The theory of optimal stopping. Houghton Mifflin, Boston (1971).

[5] Cox, D. R. and MILLER, H. D., The theory of stochastic processes, Methuen, London (1965). 
[6] Cozzocino, J. MI. and FreIfelder, L., "Cinderwriting individual drivers: A sequential approach," forthcoming in the Transactions of the Casualty Actuarial Society.

[7] DeGroor, M. H., Optimal statistical decisions, McGraw-Hill, New York (1970).

[8] Sirjaev, A. N., Statistical Sequential Analvsis (optimal stopping rules) Transactions of Mathematical Monographs, Volume 38, American IIathematical Society, Providence, Rhode Island (1973).

[9] Feller, W., "An Introduction To Probability Theory And Its Applications", vol. 2. Wiley, New York (1966).

Department of Mathematics, University of Michigan, Ann Arbor, Michigan 